\title{
Performance Evaluation of Multi Radio Integrated Navigation System under Navigation Warfare in M\&S Software
}

\author{
Heyone Kim ${ }^{1}$, Jae Hoon Son ${ }^{1}$, Sang Heon $\mathrm{Oh}^{2}$, Hyoungmin $\mathrm{So}^{3}$, and Dong-Hwan Hwang1,* \\ ${ }^{1}$ Chungnam National University, Department of Electronics Engineering, 34134 Daejeon, Korea \\ ${ }^{2}$ Navcours Co., Ltd, Integrated Navigation Division, 34014 Daejeon, Korea \\ ${ }^{3}$ Agency for Defense Development, 34186 Daejeon, Korea
}

\begin{abstract}
In this paper, a modelling and simulation software is designed in order to evaluate the performance of a multi radio integrated navigation system and the performance evaluation results are presented. The modelling and simulation software is divided into navigation algorithm module, navigation environment generation module, and graphic user interface module with performance evaluation algorithm. In order to show the validity of the design, the modelling and simulation software for GPS, KNSS, Loran-C, eLoran, and DME/VOR is implemented in C++ under Windows OS environment. Accuracy, integrity, continuity and availability are evaluated for the multi radio integrated navigation system in the modelling and simulation software. The performance evaluation results show that the designed modelling and simulation software can be effectively used for the performance evaluation of multi radio integrated navigation systems.
\end{abstract}

\section{Introduction}

The NAVWAR (NAVigation WARfare) is defined as the deliberate defensive and offensive action to assure and prevent positioning, navigation and timing information [1]. GNSS (Global Navigation Satellite System) is vulnerable to jamming and spoofing since the received signal power is very small and signal structure is open to the public [2-3]. In recent years, many research results on the alternative navigation system and anti-jamming and/or anti-spoofing receivers have been introduced to cope with these threat [4-6]. By integrating GNSS with other radio navigation system in the alternative navigation system, continuous navigation outputs can be provided in NAVWAR environments [3, 6]. As examples of these systems, integration of GPS (Global Positioning System) and ground based navigation system in U.S. (United Station) and GPS/eLoran in U.K. (United Kingdom) have been planned and carried out [7-9]. In Korea, a research on a multi radio integrated navigation system, in which GPS, KNSS (Korean Navigation Satellite System) and ground based navigation systems are integrated, have been being carried out for the outage of GPS [11-15].

Lots of tests and performance evaluations are required before the multi radio integrated navigation system is deployed for real-time navigation. For the performance evaluation, much time and cost can be saved if an M\&S S/W (Modelling and Simulation SoftWare) is used [10].

Generally, the performance of the radio navigation system is evaluated through four indices, accuracy, integrity, continuity and availability $[1,17,18]$, and relation between these four indices has pyramid scheme with the accuracy as base [18].

In this paper, the $M \& S \mathrm{~S} / \mathrm{W}$ for performance evaluation of a multi radio integrated navigation system is designed and the results of the performance evaluation are presented. The M\&S S/W is divided into navigation algorithm part, navigation environment generation part, performance evaluation part and GUI (Graphic User Interface). The designed $\mathrm{M} \& \mathrm{~S} \mathrm{~S} / \mathrm{W}$ is implemented in $\mathrm{C}$ under Windows environment. The navigation algorithm in the M\&S S/W is run for a motion of a vehicle in a normal situation, and then the performance evaluation part in the M\&S S/W is run for the outputs of the navigation algorithm, in which the performance evaluation method recommended by IMO (International Maritime Organization) is adopted. The NAVWAR effect is reflected in the navigation environment generation part and performance evaluation for the navigation algorithm in this situation is performed. The performance evaluation results are compared for two situations.

In the sequel, in section II, requirements of the $M \& S$ $\mathrm{S} / \mathrm{W}$ for performance evaluation and M\&S S/W design result to satisfy the requirements are presented. In section III, the designed M\&S S/W is implemented and performance evaluation results are presented. Finally, in section IV, concluding remarks and further studies are presented.

\footnotetext{
Corresponding author: dhhwang@ cnu.ac.kr.
} 


\section{M\&S S/W requirement and design}

\section{1. $M \& S S / W$ requirements for performance evaluation}

In order to evaluate the performance of the multi radio integrated navigation system under NAVWAR in the M\&S S/W, the M\&S S/W should satisfy the following requirements.

- The multi radio integrated navigaiton algorithms to evaluate should be prepared.

- Measurements for the multi radio integrated navigation algorithm should be prepared.

- In the measurements, motion of the vehicle should be reflected. The characteristics of the signal transmission process from signal sources to the measurments should be reflected in the measutements. The characteristics of the signal transmission can be modelled as measurements error.

- The effect of NAVWAR environment should be reflected in the M\&S S/W. Due to the NAVWAR, measurements errors becomes larger. In order to reflect the effect of NAVWAR, another measurement error is added to the generated measurements.

- The performance evaluation algorithms should be prepared in the M\&S S/W.

- In order for the user to easily set parameters of the multi radio navigaiton algorithm, measurement generation and performance evaluation and check the results of navigation algorithm and performance evalution, a GUI (Graphic User Interface) should be prepared.

\subsection{M\&S S/W design}

An M\&S S/W configuraion satisfying the requirements given above is shown in figure 1 . The M\&S S/W consists of navigation algorithm part, navigation environment generation part, performance evaluation part and GUI part. As shown in figure 1, the multi radio integrated navigation system is an integrated navigation system in which, GPS, a local satellite navigation system KNSS(Korean Navigation Satellite System) and ground based radio navigaiton systems such as Loran-C (Long range navigation), e-Loran (enhanced Loran), DME (Distance Measuring Equipment) and VOR (Very high frequency Omnidirectional Radio range). In the navigaiton environment generation part, motion of the vehicle, signal transmission characteristics and effect of NAVWAR environment are reflected.

When the M\&S S/W is implemented in a platform, the $\mathrm{S} / \mathrm{W}$ is divided into function modules as shown in figure 2. For data exchange between function modules, the message broker is included in the M\&S S/W. Due to this message broker, each function module can be independently operated. Since the functions modules are operated independently, each function module can easily modify and expand its function. The navigation algorithm module will run in a real-time multi radio integrated navigation system. Since the M\&S S/W is run based on the $M \& S$ message broker, the navigation algorithm can be ported in the real-time multi radio integrated navigation system without little modification.

In order to perform evaluation of navigaiton algorithms, the whole outputs of the navigation algorithm for one trajectory are needed, there is no data exchange of performance evaluation part with other function module. For this reason, the performnce evaluation part is included in the GUI module in the M\&S S/W.

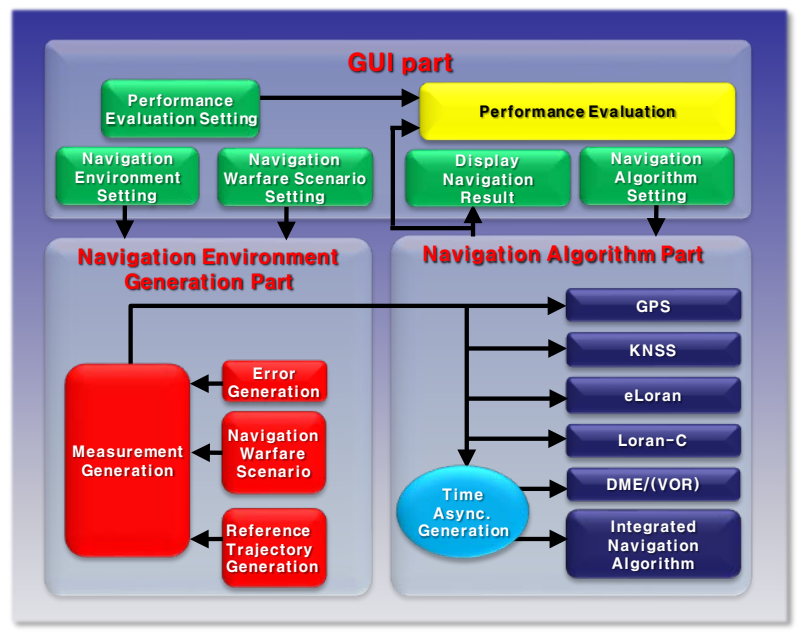

Fig. 1. M\&S S/W configuration

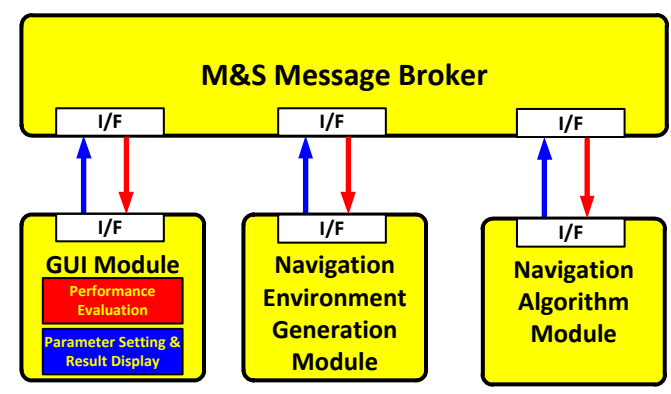

Fig. 2. Multi radio integrated navigation system M\&S S/W with message broker

\subsubsection{Navigation algorithm module}

The multi radio navigation algorithm module is shown in figure 3. Outputs of each navigation system and integrated navigation systems are calculated from the measurements. The weighted least square method and Kalman filter can be used.

\subsubsection{Navigation environment generation} module

The navigaiton environment generation module is shown in figure 4 . The navigation genertation module generates measurements for the navigaiton algorithms. It can be 
seen from figure 4 that motion of the vehicle, signal transmission characteristics and effect of NAVWAR environment are reflected in the navigation environment generation module. The visibility of the navigaiton signal sources is determined from geographic information, locations of the navigaiton signal sources and characteristics of the navigation signals.

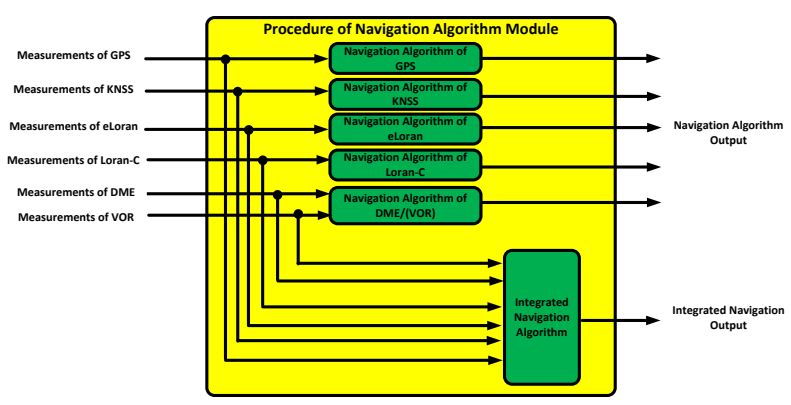

Fig. 3. Multi radio integrated navigation algorithm module

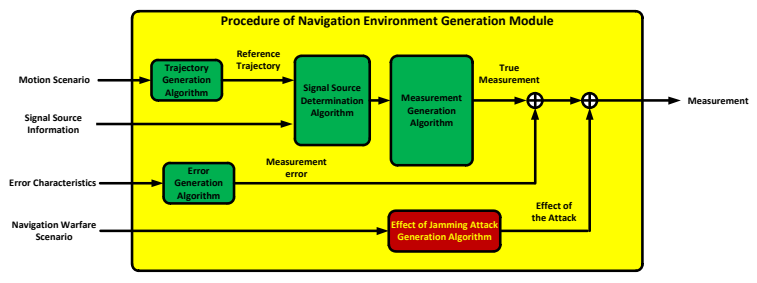

Fig. 4. Navigation environment generation module

\subsubsection{GUI module}

As shown in figure 5, GUI module receives parameters for navigation environment module, navigation algorithm module and performance evaluation module and gives navigation output display and performance evaluation result.

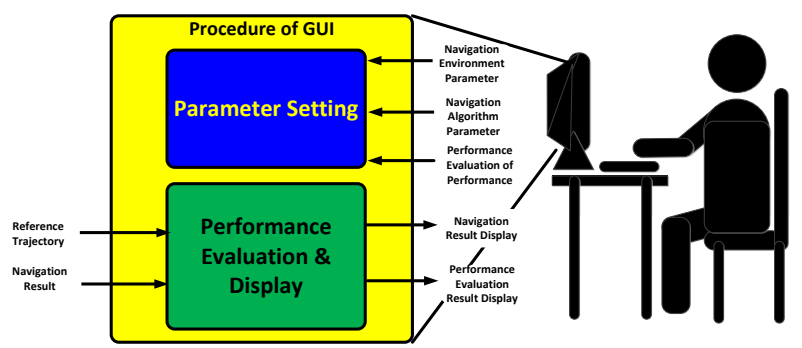

Fig. 5. GUI in the M\&S S/W

\subsubsection{Performance evaluation module}

The performance of radio navigation systems is evaluated through four indices, accuracy, integrity, continuity and availability. Relation between these four indices has pyramid scheme with the accuracy as base as shown in figure 6 [17-20].
Performacne evaluation module in the $\mathrm{M} \& \mathrm{~S} \mathrm{~S} / \mathrm{W}$ is shown in figure 7. The accuracy is evaluated from reference trajectory and navigation result. The integrity is from alarm limit and navigaion error which is result of the accuracy evaluation. The continuity is from CTI (Continuous Time Interval) and MTBF (Mean Time Between Failure) which is integrity evaluation result. The availability is from MTBF and MTTR (Mean Time To Repair) which are result of the integrity evaluation.

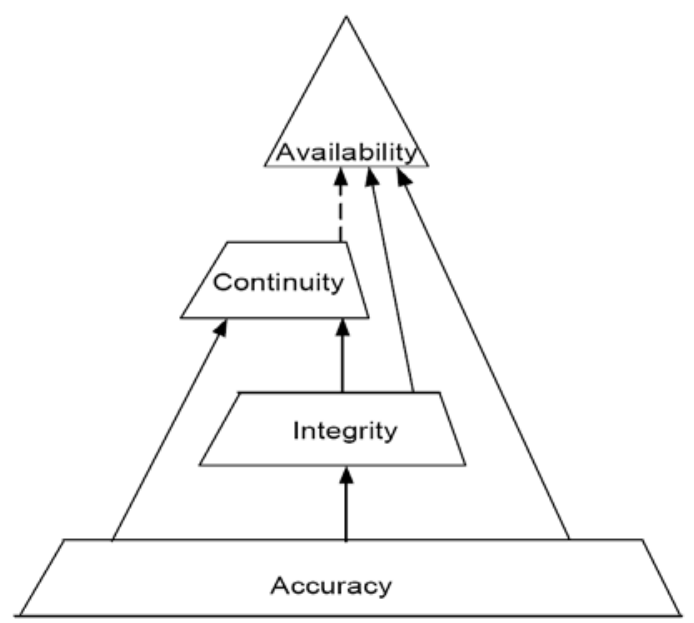

Fig. 6. Relation between four indices for performance evaluation with pyramid scheme

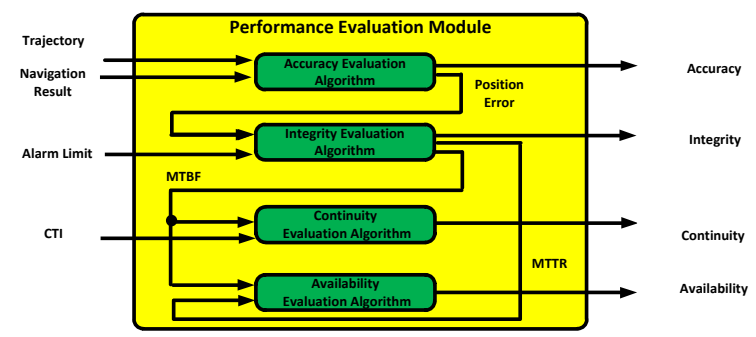

Fig. 7. Performance evaluation module

\subsubsection{Accuracy index}

The accuraccy index is the radius of distance in which navigation errors are located in 95\% probabilty. The distance is obtained from equation (1).

$$
\text { (Accuracy index })=1.96 \times R M S E
$$

where RMSE denotes Root Mean Square Error.

\subsubsection{Integrity index}

The integrity is evaluated from navigation result, Alarm Limit (AL) and Protection Level (PL). The AL is the error limit required in the navigaiton system. The PL is the estimate of error limit which is obtained from navigation output. The integrity index is given in equation (2). 
(Integrity Index $)=\frac{N_{P E<A L \& P E<P L}}{N_{\text {total }}-N_{\text {Unavailable }}} \times 100$

where $N_{P E<A L \& P E<P L}$ denotes the number of cases when PE (Position Error) is less than AL and PE less than PL. $N_{\text {total }}$ denotes all the number of cases. $N_{\text {Unavaiable }}$ denotes number of cases the when the system is not operated. In this case, $\mathrm{PL}$ is greater than $\mathrm{AL}$.

\subsubsection{Continuity index}

The continuity index is given in equation (3).

$$
\text { (Contiuity index) }=e^{-\frac{C T I}{M T B F}} \times 100
$$

In order to calculate MTBF, failure should be determined. Failure is determined to occur when PL is greater than $\mathrm{AL}$ and PE is greater than PL.

\subsubsection{Availability index}

The availability index is given in equation (4).

$$
(\text { Availability index })=\frac{M T B F}{M T B F+M T T R} \times 100
$$

The repair to calculate the MTTR is determined when AL is greater than PL and PE is less than PL.

\section{Performance evaluation}

The M\&S S/W was implemented in visual $\mathrm{C}++$ under Windows Operating System in PC. GDI(Graphic Device Interface) API(Application Programming Interface) was used for display for GUI. Performance evaluation was performed for a multi radio integrated navigation system of GPS, KNSS, eLoran, Loran-C, DME and VOR in the M\&S S/W.

\subsection{Motion of the vehicle}

Trajectory of the vehicle is shown in figure 8 . The vehicle is moving for 2500s. Initial position of the vehicle is 35.34 ${ }^{\circ}$ of latitude, $126.19^{\circ}$ of longitude and $100 \mathrm{~m}$ of altitude. After the vehicle goes up to $8 \mathrm{~km}$ of altitude, it carries 8shaped motion and descends.

\subsection{Navigation warfare environment}

In order to evaluate the performance of the navigaiton sytem in a NAVWAR environment, a jammer is located in $35.34^{\circ}$ of latitude, $125.74^{\circ}$ of longitude and $500 \mathrm{~m}$ of altitude. The effect of the jammer reflected in the navigation enenvironment generation module in the M\&S $\mathrm{S} / \mathrm{W}$. The jammer is operated during $0 \mathrm{~s}$ to $2500 \mathrm{~s}$ and power of the jammer is $30 \mathrm{~W}$. For this case, GPS signal cannot be received if the vehicle is located within $280 \mathrm{~km}$ from the jammer.

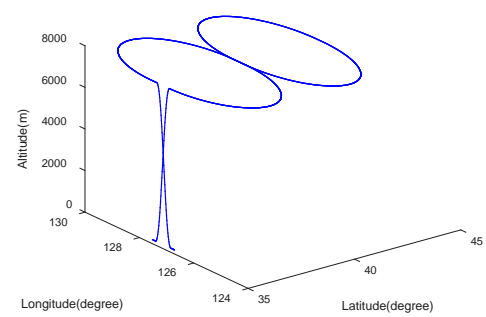

Fig. 8. Trajectory of the vehicle

\subsection{Performance evaluation result}

The performance evaluation results for the navigation algorithms is shown in table 1 when there is no jammer.

\begin{tabular}{|c|c|c|c|c|}
\hline Algorithm & $\begin{array}{c}\text { Accuracy } \\
{[\mathrm{m}]}\end{array}$ & $\begin{array}{c}\text { Integrity } \\
{[\%]}\end{array}$ & $\begin{array}{c}\text { Continuity } \\
{[\% / 15 s]}\end{array}$ & $\begin{array}{c}\text { Availability } \\
\text { [\%] }\end{array}$ \\
\hline \multirow{3}{*}{ GPS } & $\begin{array}{c}\text { North: } \\
2.815534\end{array}$ & \multirow{3}{*}{99.99} & \multirow{3}{*}{99.99} & \multirow{3}{*}{99.99} \\
\hline & $\begin{array}{c}\text { East : } \\
2.379671\end{array}$ & & & \\
\hline & $\begin{array}{c}\text { Down: } \\
3.111354\end{array}$ & & & \\
\hline \multirow{3}{*}{$\begin{array}{c}\text { Multi-radio } \\
\text { Integrated } \\
\text { Navigation } \\
\text { System } \\
\text { (GPS, KNSS, } \\
\text { eLoran, } \\
\text { Loran-C, } \\
\text { DME/VOR) }\end{array}$} & $\begin{array}{c}\text { North: } \\
2.585857\end{array}$ & \multirow{3}{*}{99.99} & \multirow{3}{*}{99.99} & \multirow{3}{*}{99.99} \\
\hline & $\begin{array}{c}\text { East : } \\
2.278709 \\
\end{array}$ & & & \\
\hline & $\begin{array}{c}\text { Down: } \\
3.047878\end{array}$ & & & \\
\hline
\end{tabular}

Table 1. Performance evaluation results with no jammer

The performance evaluation results for the navigation algorithms is shown in table 2 in the NAVWAR environment. It can be seen from table 2 that the accuracy indices are very large values in the NAVWAR enviromnent since there is intervals in which GPS signals are available. It can also seen from table 2 that the performance indes of the integrated navigation algorithms are larger than GPS navigaiton algorithm in the NAVWAR environment. Since the integrated navigaiton algorithm can provide navigation outputs even though GPS cannot. Altitude errors of output of navigation algorithms are shown in figure 8 and 9. It can also observed from figure 8 and 10 that altitude error of the integrated navigation algorithm are much less than that of GPS navigation algorithm.

\section{Concluding remarks and further studies}

In this paper, a modelling and simulation software has been designed in order to evaluate the performance of a multi radio integrated navigation system and the performance evaluation results have been presented. In 
order to show the validity of the design, the modelling and simulation software for GPS, KNSS, Loran-C, eLoran, and DME/VOR has been implemented in $\mathrm{C}++$ under Windows OS environment. Accuracy, integrity, continuity and availability have been evaluated for the multi radio integrated navigation system in a NAVWAR environment in the modelling and simulation software. The performance evaluation results showed that the designed modelling and simulation software can be effectively used for the performance evaluation of multi radio integrated navigation systems in the NAVWAR environment.

As further studies, research on integrated navigation system with an optimal performance and performance evaluation for various operation of vehicles will be performed.

This work has been supported by the National GNSS Research Center Program of Defense Acquisition Program Administration and Agency for Defense Development.

Table 2. . Performance evaluation results with a jammer

\begin{tabular}{|c|c|c|c|c|}
\hline Algorithm & $\begin{array}{c}\text { Accuracy } \\
\text { [m] }\end{array}$ & $\begin{array}{c}\text { Integri } \\
\text { ty } \\
{[\%]}\end{array}$ & $\begin{array}{c}\begin{array}{c}\text { Continuit } \\
\mathbf{y} \\
{[\% / 15 s]}\end{array} \\
\end{array}$ & 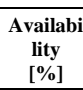 \\
\hline \multirow{3}{*}{ GPS } & North: $\infty$ & \multirow{3}{*}{84.65} & \multirow{3}{*}{92.21} & \multirow{3}{*}{78.61} \\
\hline & East : $\infty$ & & & \\
\hline & Down: $\infty$ & & & \\
\hline \multirow{3}{*}{$\begin{array}{c}\text { Multi-radio } \\
\text { Integrated } \\
\text { Navigation } \\
\text { System } \\
\text { (GPS, KNSS, } \\
\text { eLoran, } \\
\text { Loran-C, } \\
\text { DME/VOR) }\end{array}$} & $\begin{array}{c}\text { North: } \\
10.09799\end{array}$ & \multirow{3}{*}{99.99} & \multirow{3}{*}{99.99} & \multirow{3}{*}{99.99} \\
\hline & $\begin{array}{c}\text { East : } \\
6.587799\end{array}$ & & & \\
\hline & $\begin{array}{c}\text { Down: } \\
11.48838\end{array}$ & & & \\
\hline
\end{tabular}

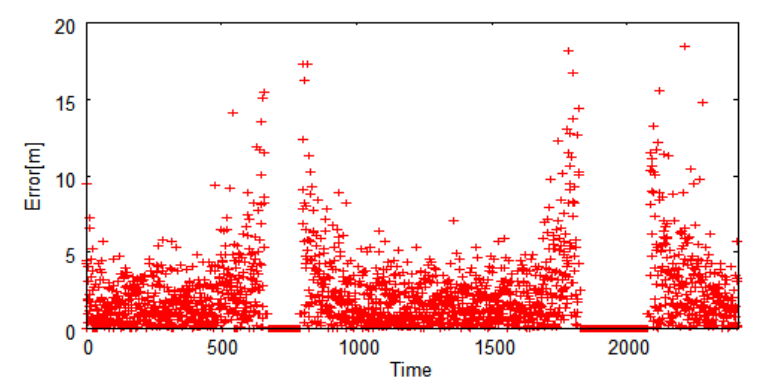

Fig. 8. Altitude error of GPS navigation algorithm

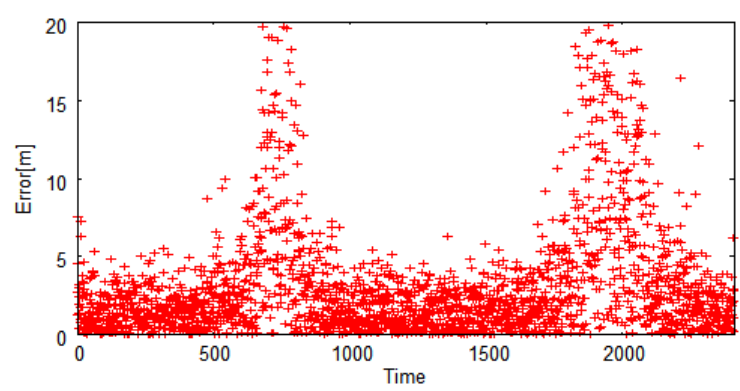

Fig. 9. Altitude error of the integrated navigation algorithm

\section{References}

1. Department of Defense, Department of Homeland Security and Department of Transportation, Federal Radio Navigation Plan 2017, pp.56-57 (2017)

2. E. D. Kaplan and C. J. Hegarty, Understanding GPS : Principles and Applications, 2nd ed. (Artech House, Norwood, MA 2006)

3. Department of Transportation, National PNT Architecture Workshop at Volpe, The Volpe Center, (2007)

4. L. Eldredge, P. Enge, M. Harrison, R. Kenagy, S. Lo, R. Loh, R. Lilly, M. Narins and R. Niles, Alternative positioning, navigation \& timing (PNT) study, Proc. of International Civil Aviation Organization Navigation Systems Panel, pp.1-19 (2010)

5. N. A. White, P. S. Maybeck and S. L. DeVilbiss, Detection of Interference/Jamming and Spoofing in a DGPS-aided Inertial System, IEEE Trans. on AES, 34, 4, pp.1208-1217 (1998)

6. S. Lo, P. Enge, F. Niles, R. Loh, L. Eldredge and M. Narins, Preliminary Assessment of Alternative Navigation Means for Civil Aviation, Proc. of ION ITM 2010, pp. 314-322 (2010)

7. P. Williams, S. Basker and N. Ward, e-Navigation and the Case for eLoran, The Journal of Navigation, 61, 3, pp. 473-484 (2008)

8. M. Koo, Y. Kim, H. So, S.H. Oh, S.C. Kim and D.H. Hwang, Modeling \& Simulation Software Design for Coverage Analysis of Multiple Radio Positioning Integration System, JPNT, 5, 2, pp. 47-57 (2016)

9. J. Lee, H. Kim, D.-H. Hwang, S. H. Oh and H. So, Multi-radio Integrated Navigation System M\&S Software Design for GNSS Back-up, Proc. of 2018 IEEE/ION PLANS, pp. 1026-1033 (2018)

10. A. Maria, Introduction to Modeling and Simulation, Proc. of 1997 Winter Simulation Conference, pp.7-13 (1997)

11. H. Kim, J. Lee, S. H. Oh, H. So, D.-H. Hwang, Design of Navigation Environment Generation Module of M\&S Software for Integrated Navigation System Performance Evaluation, JPNT, 7, 2, pp.7390 (2018)

12. C.-M. Choi, K.-S. Ko, A Study on Development Direction of Navigation System for NAVWAR, JKIICE (in Korean), 19, 3, pp. 756-763 (2015)

13. H. Kim, J. Lee, W. J. Yoo, S. H. Oh, H. K. Lee, H. So, D.-H. Hwang, Design of Navigation Environment Generation Module of Multi-radio Integrated Navigation System M\&S Software for Countermeasure to Navigation Warfare, Proc. of 2018 IEIE Summer Conference (in Korean), pp.791794 (2018)

14. S. K. Jeong, T. H. Kim, C. S. Sin and S. U. Lee, Technical trends of smart jamming for GPS signal, ETRI Electronics and Telecommunications Trends (in Korean), 27, 6, pp. 75-82 (2012) 
15. S. P. Song, M. Y. Shin, S. B. Son, Y.-B. Kim, S. J. Lee and C. Park, The Integrated eLoran/GPS Navigation Algorithm for Reduced Calculational Complexity and High Accuracy, Trans. of KIEE (in Korean), 60, 3, pp.612-619 (2011)

16. Y.-K. Lee, C.-B. Lee, S.-H. Yang, J.-K. Lee and H.D. Kong, Diurnal Effect Compensation Algorithm for a Backup and Substitute Navigation System of GPS, JKICS (in Korean), 33, 12, pp. 1225-1232 (2008)

17. H.-W. Bernhard, L. Herbert and W. Elmar, GNSS Global Navigation Satellite Systems GPS, GLONASS, Galileo, and more, (Springer, Wien, 2008)

18. European Commission 2003, World Wide Radionavigation System - Evaluation of the Galileo Performance Against Maritime GNSS Requirements, (IMO, 2003)

19. M. Tossaint, J. Samson, F. Toran and J. VenturaTraveset, The Stanford - ESA Integrity Diagram: A New tool for the user domain SBAS integrity assessment, JIN. 54, 2, pp.1-17 (2007)

20. Annual of navigation, Availability, Reliability and Continuity Model of Differential GPS Transmission, (Gdańsk Branch of the Polish Academy of Sciences, 2003) 